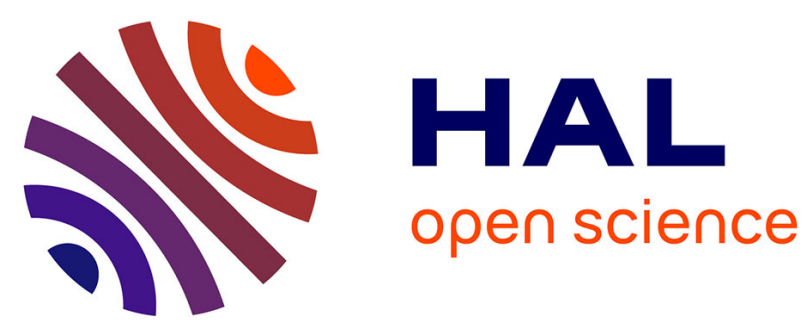

\title{
On the discontinuity of the dissipation rate associated with the temperature variance at the fluid-solid interface for cases with conjugate heat transfer
}

Cédric Flageul, Sofiane Benhamadouche, Eric Lamballais, Dominique Laurence

\section{To cite this version:}

Cédric Flageul, Sofiane Benhamadouche, Eric Lamballais, Dominique Laurence. On the discontinuity of the dissipation rate associated with the temperature variance at the fluid-solid interface for cases with conjugate heat transfer. International Journal of Heat and Mass Transfer, 2017, 111, pp.321 328. 10.1016/j.ijheatmasstransfer.2017.04.005 . hal-01504590

\author{
HAL Id: hal-01504590 \\ https://hal.science/hal-01504590
}

Submitted on 10 Apr 2017

HAL is a multi-disciplinary open access archive for the deposit and dissemination of scientific research documents, whether they are published or not. The documents may come from teaching and research institutions in France or abroad, or from public or private research centers.
L'archive ouverte pluridisciplinaire HAL, est destinée au dépôt et à la diffusion de documents scientifiques de niveau recherche, publiés ou non, émanant des établissements d'enseignement et de recherche français ou étrangers, des laboratoires publics ou privés. 


\title{
On the discontinuity of the dissipation rate associated with the temperature variance at the fluid-solid interface for cases with conjugate heat transfer.
}

\author{
Flageul Cédric ${ }^{\mathrm{a}, \mathrm{b}, *}$, Benhamadouche Sofiane ${ }^{\mathrm{b}}$, Lamballais Éric ${ }^{\mathrm{c}}$, Laurence \\ Dominique $^{\mathrm{d}, \mathrm{b}}$ \\ ${ }^{a}$ Institut Jožef Stefan, R4 Division, Jamova cesta 39, SI-1000 Ljubljana, Slovenia \\ ${ }^{b}$ EDF RED, Fluid Mechanics, Energy and Environment Dept. 6 Quai Wattier, 78401 \\ Chatou, France \\ ${ }^{c}$ Institute PPRIME, Department of Fluid Flow, Heat Transfer and Combustion, Université \\ de Poitiers, CNRS, ENSMA, Téléport 2 - Bd. Marie et Pierre Curie B.P. 30179, 86962 \\ Futuroscope Chasseneuil Cedex, France \\ ${ }^{d}$ School of Mechanical, Aerospace and Civil Engineering, The University of Manchester, \\ Sackville Street, Manchester M13 9PL, UK
}

\begin{abstract}
In the case of conjugate heat transfer, the dissipation rate associated with the temperature variance is discontinuous at the fluid-solid interface. The discontinuity satisfies a compatibility condition involving the fluid-solid thermal diffusivity and conductivity ratios and the relative contribution to the dissipation rate of its wall-normal part. The present analysis is supported by the Direct Numerical Simulations of an incompressible channel flow at a Reynolds number, based on the friction velocity, of 150, a Prandtl number of 0.71 and several values of fluid-solid thermal diffusivity and conductivity ratios.
\end{abstract}

Keywords: Conjugate heat transfer, Dissipation rate, RANS, Direct

Numerical Simulation, Turbulent channel flow

\section{Introduction}

Conjugate heat transfer describes the thermal coupling between a fluid and a solid. It is of prime importance in industrial applications where fluctuating

\footnotetext{
* Corresponding author

Email address: cedric.flageul@gmail.com (Flageul Cédric)
} 
thermal stresses are a concern, e.g. in case of a severe emergency cooling or long-term ageing of materials. For such complex applications, investigations often rely on experiments, high Reynolds RANS (Reynolds-averaged NavierStokes) or wall-modelled LES (Large Eddy Simulation). However, experimental data on conjugate heat transfer are scarce. Walls in lab rigs are often made of plexiglas and the transported scalar studied is often a dye. These common experimental configurations cannot be used to study conjugate heat-transfer as the dye does not penetrate into the wall. Analytical analysis and DNS (Direct Numerical Simulation) are valuable tools for understanding the flow physics of such complex phenomena and providing reliable data in order to improve RANS and LES modelling.

Numerical study on conjugate heat transfer started with the 2D synthetic turbulence study of Kasagi et al. ([1]). Some experimental and analytical studies have been performed prior to this study, in particular Polyakov ([2]) and Geshev ([3]), as documented by [1. The first DNS with conjugate heat transfer was a turbulent channel flow, performed by Tiselj et al. (4]). Following those studies, the authors (Flageul et al. ([5])) have also performed DNS of the turbulent channel flow with conjugate heat transfer, with a post-processing designed to produce validation data for RANS models.

The development of RANS approaches for conjugate heat transfer is relatively recent and was pioneered by Craft et al. ([6]). In order to allow an 25 accurate estimation of the fatigue, (U)RANS models adapted to conjugate heat transfer should enable the simulation of at least a few minutes of operation in realistic conditions, in order to include as much high stress amplitude events as possible, knowing they generally are low probability events (Costa Garrido et al. $([7))$.

30 The structure of the paper is as follows. In the second section, it is established that in case of conjugate heat transfer, the dissipation rate associated with the temperature variance is discontinuous at the fluid-solid interface. This discontinuity satisfies a compatibility condition involving the fluid-solid thermal diffusivity and conductivity ratios and the relative contribution to the dissipa- 
tion rate of its wall-normal part. In the third section, the case and numerical setup are described: 9 DNS of incompressible channel flow with conjugate heat transfer are presented. In the fourth section, the corresponding results are presented and the discontinuity of the dissipation rate $\varepsilon_{\theta}$ at the fluid-solid interface is highlighted. In the fifth section, our results are further discussed alongside 40 with the consequences for RANS and LES modeling.

\section{Governing equations and discontinuity of $\varepsilon_{\theta}$}

In the fluid domain $\left(\Omega_{f}\right)$, the mass and momentum equations read:

$$
\begin{aligned}
\partial_{i} u_{i} & =0 \\
\partial_{t} u_{i} & =-\frac{\partial_{j}\left(u_{i} u_{j}\right)+u_{j} \partial_{j} u_{i}}{2}-\frac{\partial_{i} p}{\rho}+\nu \partial_{j j} u_{i}+f_{i}
\end{aligned}
$$

where $\rho$ is the density, $\nu$ is the kinematic viscosity, the convective term is expressed using the skew-symetric formulation and $f_{i}$ is a source term.

In case of conjugate heat transfer, the energy equations read:

$$
\begin{aligned}
\partial_{t} T_{f} & =-\partial_{j}\left(T_{f} u_{j}\right)+\alpha_{f} \partial_{j j} T_{f}+f_{T_{f}} \text { in } \Omega_{f} \\
\partial_{t} T_{s} & =\alpha_{s} \partial_{j j} T_{s}+f_{T_{s}} \text { in } \Omega_{s} \\
T_{f} & =T_{s} \text { on } \partial \Omega_{f} \cap \partial \Omega_{s} \\
\lambda_{f} \partial_{n} T_{f} & =\lambda_{s} \partial_{n} T_{s} \text { on } \partial \Omega_{f} \cap \partial \Omega_{s}
\end{aligned}
$$

where $\Omega_{f}\left(\Omega_{s}\right), T_{f}\left(T_{s}\right), \alpha_{f}\left(\alpha_{s}\right)$ and $\lambda_{f}\left(\lambda_{s}\right)$ are the fluid (solid) domain, temperature, thermal diffusivity and thermal conductivity, respectively, $f_{T_{f}}$ and $f_{T_{s}}$ are source terms and $\partial_{n} T=\nabla(T) . \mathbf{n}$ is the wall-normal derivative of the temperature with $\mathbf{n}$ a unit vector normal to the fluid-solid interface surface $\left(\partial \Omega_{f} \cap \partial \Omega_{s}\right), \nabla$ being the gradient operator. The last 2 lines in equation (2) express the continuity of temperature and heat flux at the fluid-solid interface.

Within this context, the dissipation rate $\varepsilon_{\theta, f}\left(\varepsilon_{\theta, s}\right)$ associated with the temperature variance in the fluid (solid) domain can be defined:

$$
\begin{aligned}
\varepsilon_{\theta, f} & =2 \alpha_{f} \overline{\nabla\left(T_{f}^{\prime}\right) \cdot \nabla\left(T_{f}^{\prime}\right)} \text { in } \Omega_{f} \\
\varepsilon_{\theta, s} & =2 \alpha_{s} \overline{\nabla\left(T_{s}^{\prime}\right) \cdot \nabla\left(T_{s}^{\prime}\right)} \text { in } \Omega_{s}
\end{aligned}
$$


where $T^{\prime}$ and the overline are the fluctuating part of the temperature $T$ and the averaging operator, respectively. Using the continuity of temperature and heat flux at the fluid-solid interface, it is straightforward to show that the dissipation rates satisfy the following relation:

$$
\begin{aligned}
\frac{\varepsilon_{\theta, f}}{2 \alpha_{f}}-\frac{\varepsilon_{\theta, s}}{2 \alpha_{s}} & =\overline{\partial_{n} T_{f}^{\prime} \partial_{n} T_{f}^{\prime}}-\overline{\partial_{n} T_{s}^{\prime} \partial_{n} T_{s}^{\prime}} \text { on } \partial \Omega_{f} \cap \partial \Omega_{s} \\
& =\overline{\partial_{n} T_{f}^{\prime} \partial_{n} T_{f}^{\prime}}\left[1-\left(\frac{\lambda_{f}}{\lambda_{s}}\right)^{2}\right] \text { on } \partial \Omega_{f} \cap \partial \Omega_{s}
\end{aligned}
$$

Using the thermal properties $\alpha$ and $\lambda$, dimensionless numbers can be derived. Following Flageul et al. ([5]), one defines $G$ as the fluid-to-solid thermal diffusivity ratio and $G_{2}$ as the solid-to-fluid thermal conductivity ratio:

$$
G=\frac{\alpha_{f}}{\alpha_{s}} \quad, \quad G_{2}=\frac{\lambda_{s}}{\lambda_{f}}
$$

Combining $G$ and $G_{2}$, one may obtain the thermal activity ratio $K\left(\frac{1}{K}=G_{2} \sqrt{G}\right)$ as defined by Geshev ([3]) and Tiselj et al. ([4]), which is also the fluid-tosolid thermal effusivity ratio. On this basis, equation (4), combined with the definition of $\varepsilon_{\theta, f}$ in equation 3 turns to:

$$
\begin{aligned}
1-G \frac{\varepsilon_{\theta, s}}{\varepsilon_{\theta, f}} & =\frac{\overline{\partial_{n} T_{f}^{\prime} \partial_{n} T_{f}^{\prime}}}{\overline{\nabla T_{f}^{\prime} \cdot \nabla T_{f}^{\prime}}}\left[1-\frac{1}{G_{2}^{2}}\right] \\
\Longleftrightarrow \frac{1}{G}-\frac{\varepsilon_{\theta, s}}{\varepsilon_{\theta, f}} & =\frac{\overline{\partial_{n} T_{f}^{\prime} \partial_{n} T_{f}^{\prime}}}{\overline{\nabla T_{f}^{\prime} \cdot \nabla T_{f}^{\prime}}}\left[\frac{1}{G}-K^{2}\right] \\
\Longleftrightarrow \frac{\varepsilon_{\theta, s}}{\varepsilon_{\theta, f}} & =\frac{\overline{\partial_{n} T_{f}^{\prime} \partial_{n} T_{f}^{\prime}}}{\overline{\nabla T_{f}^{\prime} \cdot \nabla T_{f}^{\prime}}} K^{2}+\left(1-\frac{\overline{\partial_{n} T_{f}^{\prime} \partial_{n} T_{f}^{\prime}}}{\overline{\nabla T_{f}^{\prime} \cdot \nabla T_{f}^{\prime}}}\right) \frac{1}{G}
\end{aligned}
$$

65 It is important to stress that $\mathbf{n}$ is locally well-defined as long as the fluid-solid interface surface is flat or curved but becomes ill-defined for instance at the edge of a corner. Therefore, in case of conjugate heat transfer, the dissipation rate $\varepsilon_{\theta}$ at the fluid-solid interface satisfies the compatibility condition (6) for any smooth interface.

In the following, any ratio $\frac{\varepsilon_{\theta, s}}{\varepsilon_{\theta, f}} \neq 1$ corresponds to a discontinuity of the the dissipation rate $\varepsilon_{\theta}$ across the fluid-solid interface. It is important to stress 
that the relative contribution of the wall-normal part in $\varepsilon_{\theta, f}$ is bounded in $[0,1]$. Therefore, equation (6) is a convex combination between $\frac{1}{G}$ and $K^{2}$.

On the one hand, if the conjugate case is close to an imposed temperature one

75 (conducting solid, $G_{2} \gg 1$ ), then the wall-normal contribution in $\varepsilon_{\theta, f}$ dominates at the interface and the discontinuity scales with the squared thermal activity ratio $K$. On the other, if the conjugate case is close to an imposed heat flux one (insulating solid, $G_{2} \ll 1$ ), then the wall-parallel contribution in $\varepsilon_{\theta, f}$ dominates at the interface and the discontinuity scales with the inverse of the thermal diffusivity ratio $G$. For the other cases, the discontinuity is bounded by $\frac{1}{G}$ and $K^{2}$. This range may be quite extended, for instance, considering pressurized water as the fluid and steel as the solid, approximate values are $K^{2} \approx 0.01$ and $1 / G \approx 60$.

\section{Case and numerical setup}

Present simulations are based on the open-source software Incompact3d developed at Université de Poitiers and Imperial College London by Laizet et al. $([8,[9])$. Sixth-order compact schemes are used on a Cartesian grid stretched in the wall-normal direction. The pressure is computed with a direct solver on a staggered grid while velocity components and temperature are collocated.

In the present study, $x, y$ and $z$ stand for the streamwise, wall-normal and spanwise directions, respectively, as sketched in figure 1. Periodic boundary conditions are used in the streamwise and spanwise directions. The source term driving the channel flow is present only in the streamwise direction: it is a constant in space and time fitted so that the averaged bulk velocity is 1 . This source term physically represents the mean pressure gradient compensating the viscous friction at the wall in order to reach a statistically steady state. The channel half-height is also 1, and the Reynolds number based on those quantities is 2280 , while the Prandtl number is 0.71 and the density is 1 .

The main simulation parameters are recalled in table 1 and compared with reference ones (Kasagi et al. ([10]) and Tiselj et al. ([4)). As described in 


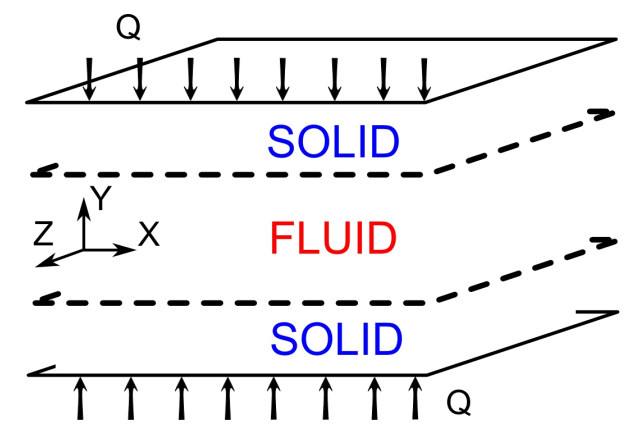

Figure 1: Sketch of the computational domain.

Flageul et al. ([5]), the scalar diffusion scheme used is $4^{\text {th }}$ order accurate in the streamwise direction and $6^{\text {th }}$ order accurate in the others. Compared to the simulation from Kasagi et al. ([10]), our domain is $63 \%$ longer, $35 \%$ wider while we use cells of a similar size. In addition, the duration of our simulation is almost 14 times longer while our time step is 6 times smaller. This point is further discussed in the appendix A. It is the result of a trade-off between the necessity to represent large scale fluctuations, with a long lifetime, which are present deep inside the solid domain and the smaller fluctuations, with a shorter lifetime, which are present in the fluid and are related to the distinctive intermittent character of the passive scalar, as reported by Galantucci and Quadrio ([1]).

The scalar conservation equation in the fluid domain contains a source term proportional to the streamwise velocity, as defined by Kasagi et al. ([10]). If we consider an infinite channel flow constantly heated, the bulk temperature increases linearly with $x$. A change of variable is used, to compensate this linear increase and to allow periodicity in the streamwise direction. This gives rise to the source terms: $f_{T_{f}}=\alpha_{f} u_{x}$ and $f_{T_{s}}=0$. The similar case of a channel flow constantly heated at one wall and cooled at the other which does not give rise to a source term was studied firstly by Kim and Moin ([12]). As it was established by Kawamura et al. [13] that both cases present similar statistics in the near-wall region, this heat source/sink term has no effect on the statistics around the fluid-solid interface. 
The case and simulation setup are similar to the ones detailed in [5], except for the thermal properties ratios $G$ and $G_{2}$. The thickness of the top and bottom walls is half the thickness of the fluid domain. Tiselj et al. (4) performed similar simulations using solid domains at least 3 times thinner and reported that as the wall get thinner, the conjugate cases get closer to the imposed heat flux one. This is the natural behaviour when the boundary condition at the outer wall is an imposed heat flux, as in their study and herein. In this study, we consider that the solid domain is thick enough for the boundary condition imposed at the outer wall to have no significant impact on the statistics at the fluid-solid interface.

In the present study, the DNS performed with conjugate heat transfer are labelled $\mathrm{CHT}_{i j}$. As indicated in table 2 , the index $i$ and $j$ stand for the ratio of thermal diffusivity and conductivity, respectively. The indexes can be equal to 0,1 or 2 , the corresponding thermal properties ratios being $0.5,1$ and 2, respectively. The results obtained with conjugate heat transfer are compared with the non-conjugate cases of locally imposed temperature (isoT) and locally imposed heat flux $(i s o Q)$ at the fluid boundary.

\begin{tabular}{|l|c|c|c|}
\hline & Present & Kasagi et al. ([10]) & Tiselj et al. ([4] $)$ \\
\hline Domain & {$[25.6,2,8.52]$} & {$[5 \pi, 2,2 \pi]$} & {$[5 \pi, 2, \pi]$} \\
\hline Grid & {$[256,193,256]$} & {$[128,97,128]$} & {$[128,97,65]$} \\
\hline$R e_{\tau}$ & 149 & 150 & 150 \\
\hline$\Delta y^{+}$ & {$[0.49,4.8]$} & {$[0.08,4.9]$} & {$[0.08,4.9]$} \\
\hline$\left[\Delta_{x}^{+}, \Delta_{z}^{+}\right]$ & {$[14.8,5.1]$} & {$[18.4,7.36]$} & {$[18.4,7.4]$} \\
\hline$\Delta t^{+}$ & 0.02 & 0.12 & 0.12 \\
\hline Duration & 29000 & 2100 & 6000 \\
\hline
\end{tabular}

Table 1: Simulation parameters. 


\begin{tabular}{|c|c|c|c|c|}
\hline & \multicolumn{3}{|c|}{$G_{2}$} \\
\hline & & 0.5 & 1 & 2 \\
\hline \multirow{3}{*}{$G$} & 0.5 & $C H T_{00} / 8$ & $C H T_{01} / 2$ & $C H T_{02} / 0.5$ \\
\hline & 1 & $C H T_{10} / 4$ & $C H T_{11} / 1$ & $C H T_{12} / 0.25$ \\
\hline & 2 & $\mathrm{CHT}_{20} / 2$ & $\mathrm{CHT}_{21} / 0.5$ & $\mathrm{CHT}_{22} / 0.125$ \\
\hline
\end{tabular}

Table 2: Case labels and associated squared thermal activity ratio $C H T_{i j} / K^{2}$ depending on the thermal properties ratios $G$ and $G_{2}$.

\section{Results}

For the channel flow configuration, at the present Reynolds and Prandtl numbers, it is established (Tiselj et al. (4]), Flageul et al. ([5])) that the boundary condition used for the passive scalar has an impact in the fluid domain limited to the vicinity of the wall $\left(y^{+}<20\right)$. In the following, the fluid-solid interface is located at $y^{+}=0$, the fluid (solid) domain corresponding to $y^{+}>0$

${ }_{145}\left(y^{+}<0\right)$. As we focus on the discontinuity of $\varepsilon_{\theta}$ at the fluid-solid interface, the present results are plotted only for $-10<y^{+}<10$.

In figure 2 (top row), the temperature variance is continuous across the interface. This is a direct consequence of the continuity of the instantaneous temperature at the interface. However, the derivative of the temperature variance can be discontinuous across the interface. Using the continuity of the heat flux across the interface, one obtains:

$$
\partial_{y} T_{f}^{\prime 2}=G_{2} \partial_{y} T_{s}^{\prime 2}
$$

For the present results, the discontinuity in the slope of the temperature variance at the fluid-solid interface is clearly visible for the case $\mathrm{CHT}_{22}$. Compared with the $i s o T$ and $i s o Q$ cases, for the conjugate cases studied, in the fluid domain, the higher $G$ or $G_{2}$, the closer to the isoT case.

This simple trend, can not be transposed to the solid domain: one can notice that the temperature variance curves cross each other in the solid domain for some of the cases. For instance, cases $\mathrm{CHT}_{21}$ and $\mathrm{CHT}_{02}$ cross around $y^{+} \approx-2$. 
This highlights the complex behaviour of the present conjugate cases where the cases with different fluid-solid properties ratios lead to similar dissipation rates in the fluid domain, but not in the solid one. It is also observed that, for a given thermal activity ratio $K$, the relative contribution of the wall-normal part in 

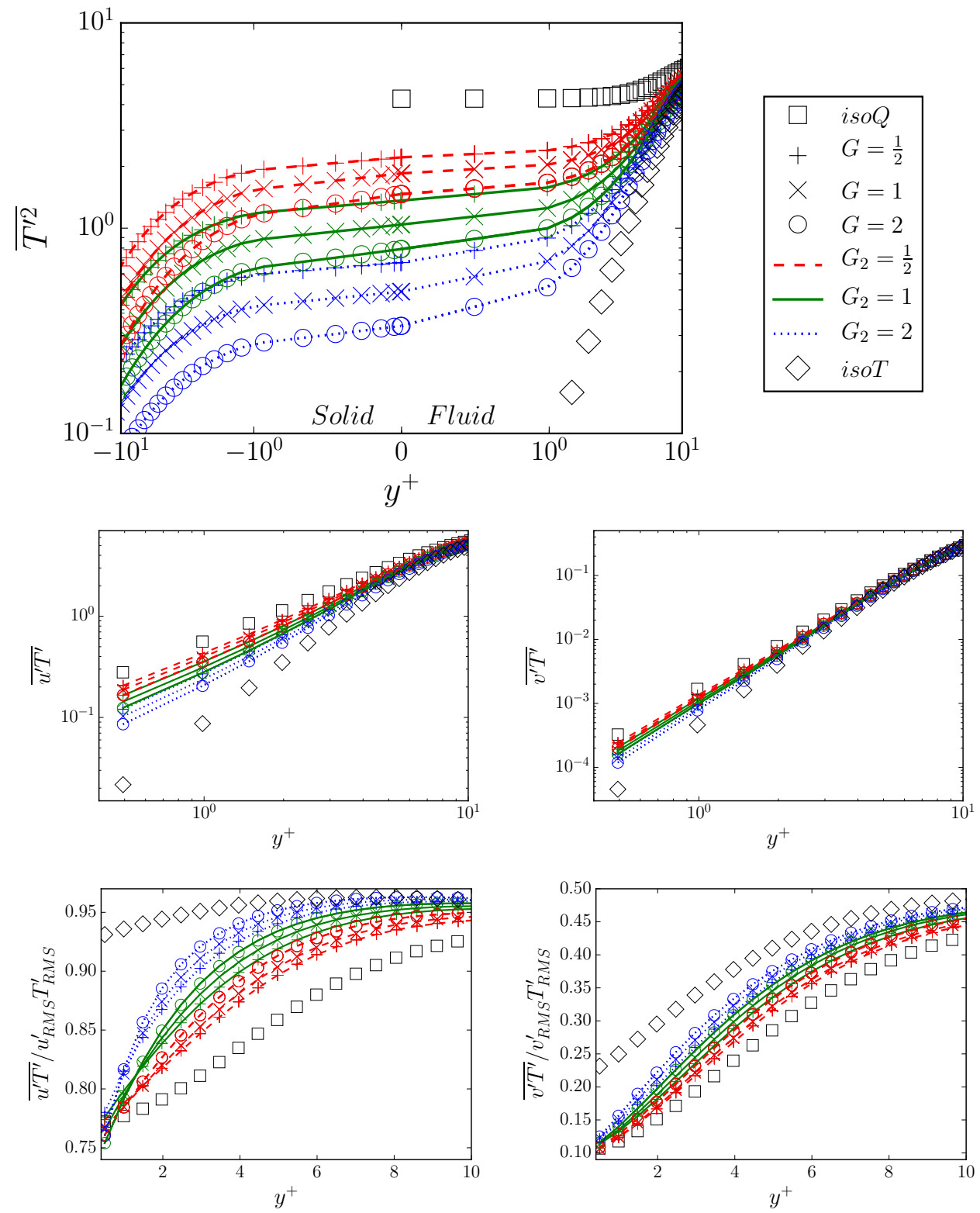

Figure 2: Top: Temperature variance. Logarithmic scaling for $\overline{T^{\prime 2}}$ and $y^{+}$, except for $-1<$ $y^{+}<1$ : linear scaling for $y^{+}$. Middle: Turbulent heat fluxes. Bottom: One-point correlation coefficients associated with the turbulent heat fluxes. 

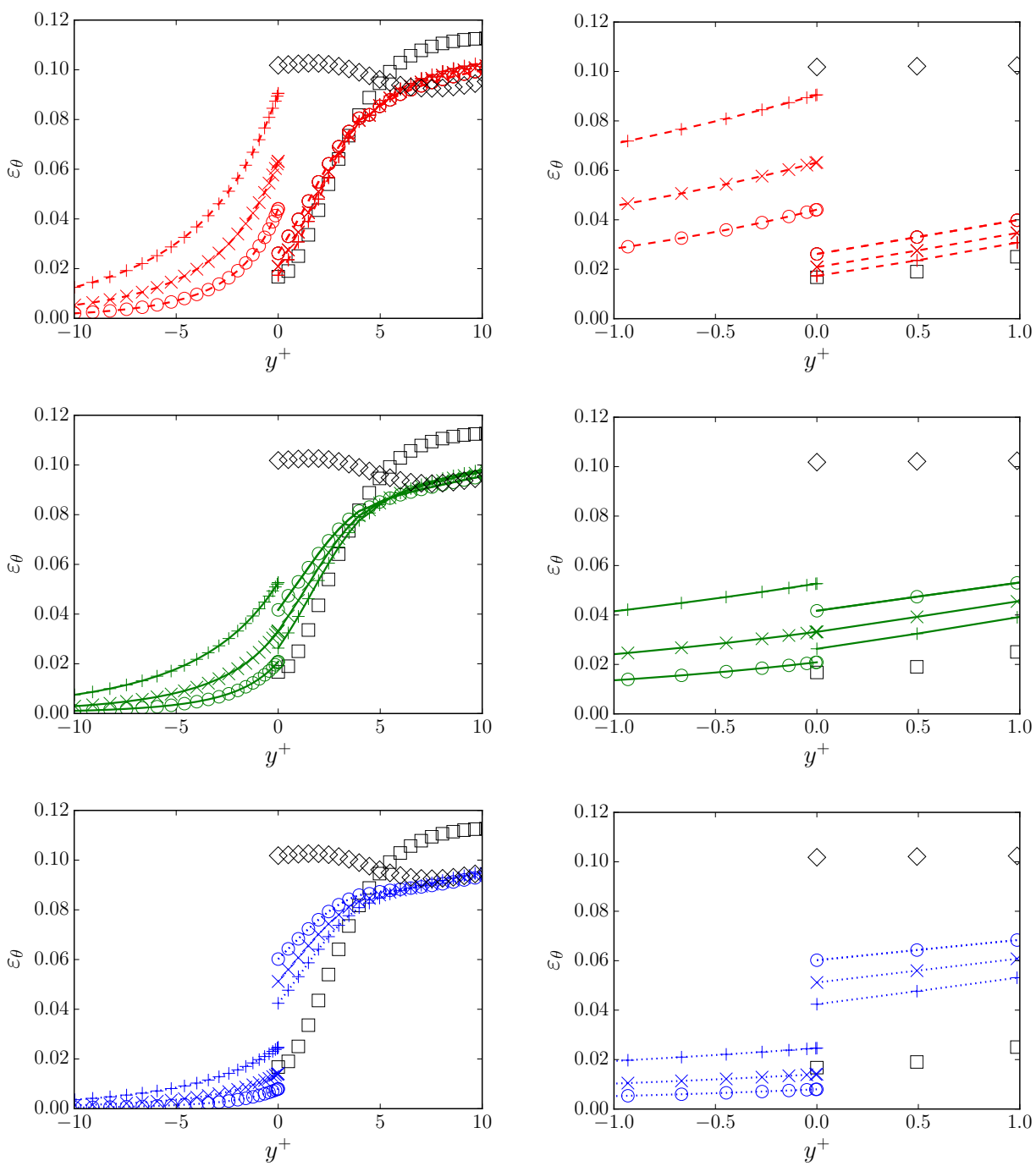

Figure 3: Dissipation rate $\varepsilon_{\theta}$ around the fluid-solid interface. Lines and symbols as in figure2 Top: $G_{2}=0.5$. Middle : $G_{2}=1$. Bottom : $G_{2}=2$ 

for conjugate cases with $G$ and $G_{2}$ around unity, it seems that, in the fluid domain, the amplitude of the fluctuating temperature gradient depends only on the thermal activity ratio $K$ while the associated anisotropy has a more complex behaviour. This behaviour, combined with different thermal properties ratios, lead to different dissipation rates in the solid domain for a given thermal activity ratio $K$.

In figure 5, the relative contribution of the wall-normal part in $\varepsilon_{\theta, f}$ is plotted. For this relative contribution, the global trend observed in figure 2 is well recovered: in the fluid domain, the higher $G$ or $G_{2}$, the closer to the isoT case. At

\section{Discussion}

The DNS performed show an agreement with equation (6) within the statistical uncertainty, as shown in table 3 . The complex behaviour of the present conjugate cases has its roots in this equation and in the relative contribution of the wall-normal part in $\varepsilon_{\theta, f}$. This quantity is related to the anisotropy of the fluctuating temperature gradient at the wall, a quantity that is not easily accessible for most of the (U)RANS models and LES wall models. The present analytical analysis and the accompanying simulations results could provide a solid ground for building new turbulence models better suited for cases with conjugate heat transfer.

Prospects on RANS models for conjugate heat transfer by the authors and co-workers have shown that building a model for the temperature variance and the associated dissipation rate in the solid domain is feasible following Craft et 

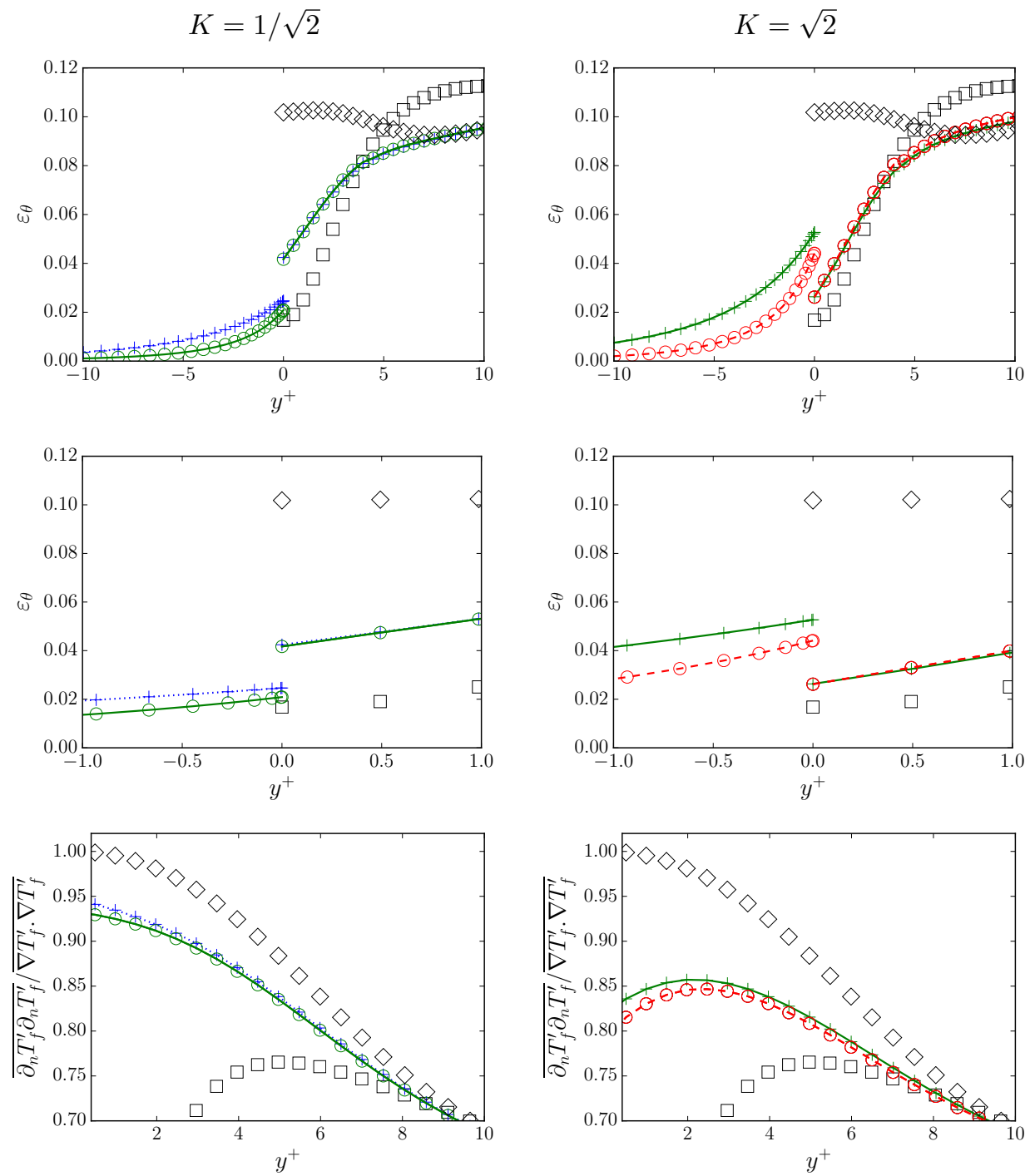

Figure 4: Dissipation rate $\varepsilon_{\theta}$ around the fluid-solid interface (top and middle) and relative contribution of the wall-normal part in $\varepsilon_{\theta, f}$ (bottom). Lines and symbols as in figure 2 Left: $K=1 / \sqrt{2}$. Right $: K=\sqrt{2}$ 


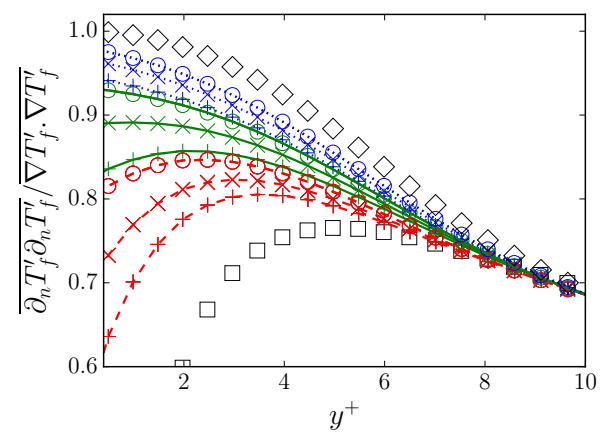

Figure 5: Relative contribution of the wall-normal part in the dissipation rate $\varepsilon_{\theta, f}$. Lines and symbols as in figure 2

\begin{tabular}{c|c|c|c|c|}
\multicolumn{4}{c}{} & \multicolumn{3}{c|}{$G_{2}$} \\
\cline { 2 - 5 } \multicolumn{1}{c|}{} & 0.5 & 1 & 2 \\
\hline \multirow{2}{*}{$G .5$} & $5.2(2.99 \% 0$ & $2.0(1.01 \% 0$ & $0.58(0.75 \% 0)$ \\
\hline 1 & $3.0(2.24 \% 0)$ & $1.0(1.19 \% 0$ & $0.27(0.78 \% 0)$ \\
\hline 2 & $1.68(1.68 \% 0)$ & $0.50(1.01 \% 0)$ & $0.13(0.70 \% 0)$ \\
\hline
\end{tabular}

Table 3: Computed ratio $\varepsilon_{\theta, s} / \varepsilon_{\theta, f}$ at the fluid-solid interface accompanied by $10^{3}$ times the relative error between the computed and expected values (expected value obtained using computed $\overline{\partial_{n} T_{f}^{\prime} \partial_{n} T_{f}^{\prime}} / \overline{\nabla T_{f}^{\prime} . \nabla T_{f}^{\prime}}$ and equation 6, ) 
al. 6]. However, much more work remains to be done to model the relative contribution of the wall-normal part in $\varepsilon_{\theta, f}$ so as to correctly handle the discontinuity of $\varepsilon_{\theta}$ at the fluid-solid interface. As suggested by Wu et al. [15, a workaround could reside in scalar integral length scales. Following Donzis et al. [16], they propose a two-point length scale which is continuous across the fluidsolid interface. Even though a value for $\varepsilon_{\theta}$ could be derived from this length scale, this is only a preliminary sketch, and there is currently no RANS model ready for fluid-solid thermal coupling.

\section{Conclusion}

An analytical analysis of cases with conjugate heat transfer has been performed and a compatibility condition at the fluid-solid interface have been ex-

the intermittency of the scalar, characterized by a strong coupling between large and small scales. Those considerations, combined with the discontinuity of $\varepsilon_{\theta}$ at the fluid-solid interface established here, sketch a modelling challenge, within reach of experimentalists and simulationists (Sreenivasan et al. 20, Johansson 245 et al. 21, Germaine et al. 22]).

Regarding subgrid-scale (SGS) models and wall-models for LES: even for wall-resolved LES, asymptotic analysis shows that the existence of temperature 
fluctuations at the wall discards any SGS model based on a constant turbulent Prandtl number for temperature. Thus, simpler SGS models for LES, like the combination of the WALE model for momentum and a constant turbulent Prandtl number around unity for temperature, often encountered in semiacademic and industrial applications, may be flawed for conjugate heat-transfer cases.

This theoretical flaw is not specific to conjugate heat-transfer as the dynamics of a passive scalar differs from the dynamics of velocity (Sagaut, ([23])). It does not prevent LES based on simpler SGS models from providing reasonably good estimations for quantities like the averaged temperature, its variance, the turbulent heat fluxes, or even the spectrum of the temperature. Thus, the ability of LES SGS models and wall-models to investigate configurations with conjugate heat-transfer should be carefully examined.

The thermal properties ratio $G$ and $G_{2}$ are close to unity in the present work. A limiting behaviour close to isoT or $i s o Q$ should be reached for ratios further away from unity. However, it must be stressed that for DNS, statistical convergence is difficult to reach when $\operatorname{Pr} \ll 1$ or $\operatorname{Pr} \gg 1$ : very long domains or very fine grids for the temperature equation have to be used. Similar measures may be necessary for simulations with ratios of fluid-solid thermal properties further away from unity.

For the channel flow configuration, it would be very interesting to obtain scalings for the discontinuity: how does it depends on the Reynolds number, on the Prandtl number and on the fluid-solid thermal properties ratios when they are further away from unity? Last but not least, it would be exciting to study the ability of wall-resolved LES to compute that discontinuity. This would allows us to build a database for the discontinuity that includes higher Reynolds number and complex cases, with the hope to help the development of specific 275 (U)RANS models or LES wall-models adapted to conjugate heat-transfer.

Source code and data associated with the present paper are available at http://dx.doi.org/10.17632/3c7v3cnwvg.1 and https://repo.ijs.si/CFLAG/ incompact3d under the GNU GPL v3 licence. 


\section{Acknowledgements}

280 for funding the present study (CIFRE 2012/0047) and providing computational time on Zumbrota supercomputer (IBM - Blue-geneQ).

\section{Appendix A. Statistical uncertainty}

In CFD, the finite extension of computational domains in space and time

and the limited number of cells used to discretize the domain lead to statistical uncertainty. This uncertainty depends on the variable considered. A relatively coarse grid often lead to a good estimation of the averaged velocity but to a bad estimation of Reynolds stresses.

For the turbulent channel flow configuration at $R e_{\tau}=160$, considering a passive scalar subjected to a Dirichlet boundary condition with $\operatorname{Pr}=1$, using a pseudo-spectral method, Galantucci and Quadrio ([11]) have established that the accurate estimation of $\varepsilon_{\theta}$ in the buffer layer $\left(10<y^{+}<30\right)$ requires a very fine grid $\left(\Delta_{x}^{+}=\Delta_{z}^{+}=1\right.$ and $\left.0.4<\Delta_{y}^{+}<2\right)$. Fortunately, near the wall $\left(y^{+}<7\right)$, the grid requirement is less stringent and their simulation on the very fine grid matches the simulation performed on a more classical grid $\left(\Delta_{x}^{+}=10\right.$, $\Delta_{z}^{+}=5$ and $\left.0.9<\Delta_{y}^{+}<4\right)$.

In Flageul et al. ([5]), this mesh requirement was assessed and we showed that the present grid was slightly too coarse for the scalar as we observed an over-estimation of $\varepsilon_{\theta}$ in the buffer-layer. We also showed that the present grid, combined with some extra numerical dissipation applied only on the scalar, only in the streamwise direction and only at high frequencies (using implicit Spectral Vanishing Viscosity, see Lamballais et al. (24])) allowed an accurate estimation of $\varepsilon_{\theta}$. Strictly speaking, this is valid only for the case studied there which uses the same thermal properties in the fluid and solid domains. In the present paper, it is considered that the same strategy can be applied to cases

with ratios of thermal properties close to unity providing a good estimation of $\varepsilon_{\theta}$ near the fluid-solid interface. 
Another source of uncertainty for statistics is the size of the domain in case of homogeneous directions associated with periodic boundary conditions. For the around the fluid-solid interface.

It must be acknowledged that our simulations were instrumented to obtain the budget of $\varepsilon_{\theta}$ but also the budget of the dissipation rates associated with 


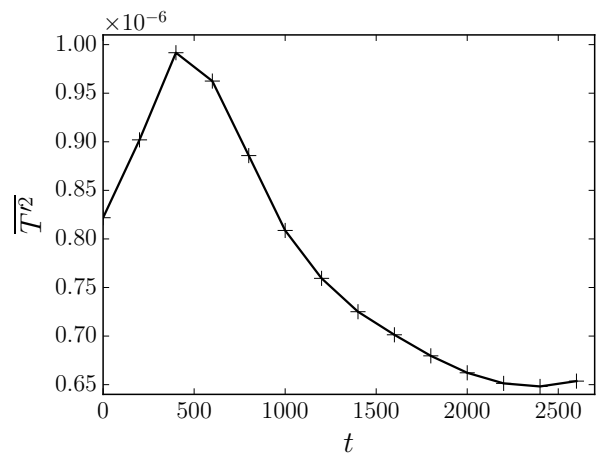

Figure A.6: Convergence during the simulation of $\overline{T^{\prime 2}}$ at the outer wall for the case $C H T_{11}$. Time $t$ and $\overline{T^{\prime 2}}$ are in computational units.

the turbulent heat fluxes. However, the present grid is probably too coarse and those budgets are not well balanced. This is why they are not presented here. Considering the relatively coarse grid used, the time step may appear unnecessary small. It seems important to recall that in our study, the fluid and solid solvers are distinct and weakly coupled, as reported in [5]. Verstraete and Scholl ([25]) have established that such coupling strategies are subjected to stability constraints. They showed that the efficiency and the accuracy of the coupled solver may be lowered when the stability criterion is hardly met.

Within this framework, the small time step used was a guarantee of temporal accuracy. While lower accuracy was the drawback of the weak coupling developed, it allowed us to build a plug-and-play module for fluid-solid thermal coupling. This module is now used to study more complex semi-academic configurations with conjugate heat-transfer like the jet in crossflow (Wu et al. ([26])) and the impinging jet ( Dairay et al. ([27])).

For further investigations of the turbulent channel flow with conjugate heattransfer, a pseudo-spectral code able to handle several passive scalars with si350 multaneous solving of the fluid and solid temperature, such as the one in [4, would probably be better fitted. 


\section{References}

[1] N. Kasagi, A. Kuroda, M. Hirata, Numerical investigation of near-wall turbulent heat transfer taking into account the unsteady heat conduction

in the solid wall, Journal of Heat Transfer 111 (2) (1989) 385-392. doi: $10.1115 / 1.3250689$

[2] A. Poliakov, Wall effect on temperature fluctuations in the viscous sublayer, High Temperature Science 12 (1974) 286-293.

[3] P. Geshev, Influence of heat conduction of the wall on the turbulent prandtl number in the viscous sublayer, Journal of engineering physics 35 (2) (1978) 949-952. doi:10.1007/BF00860218.

[4] I. Tiselj, R. Bergant, B. Mavko, I. Bajsić, G. Hetsroni, Direct numerical simulation of turbulent heat transfer in channel flow with heat conduction in the solid wall, Journal of heat transfer 123 (5) (2001) 849-857. doi: $10.1115 / 1.1389060$

[5] C. Flageul, S. Benhamadouche, É. Lamballais, D. Laurence, Dns of turbulent channel flow with conjugate heat transfer: Effect of thermal boundary conditions on the second moments and budgets, International Journal of Heat and Fluid Flow 55 (2015) 34-44. doi:10.1016/j.ijheatfluidflow. 2015.07 .009

[6] T. J. Craft, H. Iacovides, S. Uapipatanakul, Towards the development of rans models for conjugate heat transfer, Journal of Turbulence (11) (2010) N26. doi:10.1080/14685248.2010.494608.

[7] O. C. Garrido, S. El Shawish, L. Cizelj, Uncertainties in the thermal fatigue 375 assessment of pipes under turbulent fluid mixing using an improved spectral

口 loading approach, International Journal of Fatigue 82 (2016) 550-560. doi: 10.1016/j.ijfatigue.2015.09.010

[8] S. Laizet, E. Lamballais, High-order compact schemes for incompressible flows: A simple and efficient method with quasi-spectral accuracy, Journal 
of Computational Physics 228 (16) (2009) 5989 - 6015. doi:10.1016/j. jcp.2009.05.010.

[9] S. Laizet, N. Li, Incompact3d: A powerful tool to tackle turbulence problems with up to $\mathrm{o}\left(10^{5}\right)$ computational cores, International Journal for $\mathrm{Nu}-$ merical Methods in Fluids 67 (11) (2011) 1735-1757. doi:10.1002/fld. 2480.

[10] N. Kasagi, Y. Tomita, A. Kuroda, Direct numerical simulation of passive scalar field in a turbulent channel flow, Journal of heat transfer 114 (3) (1992) 598-606. doi:10.1115/1.2911323.

[11] L. Galantucci, M. Quadrio, Very fine near-wall structures in turbulent scalar mixing, International Journal of Heat and Fluid Flow 31 (4) (2010) 499-506. doi:10.1016/j.ijheatfluidflow.2010.04.002

[12] J. Kim, P. Moin, Transport of passive scalars in a turbulent channel flow, in: Turbulent Shear Flows 6, Springer, 1989, pp. 85-96. doi:10.1007/ 978-3-642-73948-4_9.

[13] H. Kawamura, H. Abe, K. Shingai, Dns of turbulence and heat transport in a channel flow with different reynolds and prandtl numbers and boundary conditions, Turbulence, Heat and Mass Transfer 3 (2000) 15-32.

[14] P. Orlandi, D. Sassun, S. Leonardi, Dns of conjugate heat transfer in presence of rough surfaces, International Journal of Heat and Mass Transfer 100 (2016) 250-266. doi:10.1016/j.ijheatmasstransfer.2016.04.035

[15] Z. Wu, D. Laurence, H. Iacovides, I. Afgan, Direct simulation of conjugate heat transfer of jet in channel crossflow, International Journal of Heat and Mass Transfer 110 (2017) 193-208. doi:10.1016/j.ijheatmasstransfer. 2017.03 .027

[16] D. Donzis, K. Sreenivasan, P. Yeung, Scalar dissipation rate and dissipative anomaly in isotropic turbulence, Journal of Fluid Mechanics 532 (2005) 199-216. doi:10.1017/S0022112005004039. 
[17] A. N. Kolmogorov, The local structure of turbulence in incompressible viscous fluid for very large reynolds numbers, in: Dokl. Akad. Nauk SSSR, Vol. 30, JSTOR, 1941, pp. 301-305.

[18] K. Sreenivasan, On local isotropy of passive scalars in turbulent shear flows, in: Proceedings of the Royal Society of London A: Mathematical, Physical and Engineering Sciences, Vol. 434, The Royal Society, 1991, pp. 165-182. doi:10.1098/rspa.1991.0087.

[19] Z. Warhaft, Passive scalars in turbulent flows, Annual Review of Fluid 口 Mechanics 32 (1) (2000) 203-240. doi:10.1146/annurev.fluid.32.1. 203.

[20] K. Sreenivasan, R. Antonia, H. Danh, Temperature dissipation fluctuations in a turbulent boundary layer, The Physics of Fluids 20 (8) (1977) 12381249. doi:10.1063/1.862005.

[21] A. V. Johansson, P. M. Wikström, Dns and modelling of passive scalar transport in turbulent channel flow with a focus on scalar dissipation rate

(1) modelling, Flow, turbulence and combustion 63 (1-4) (2000) 223. doi: 10.1023/A : 1009948606944

[22] E. Germaine, L. Mydlarski, L. Cortelezzi, Evolution of the scalar dissipation rate downstream of a concentrated line source in turbulent channel flow,

口 Journal of Fluid Mechanics 749 (2014) 227-274. doi:10.1017/jfm.2014. 170.

[23] P. Sagaut, Large eddy simulation for incompressible flows: an introduction, Springer Science \& Business Media, 2006.

[24] É. Lamballais, V. Fortuné, S. Laizet, Straightforward high-order numerical dissipation via the viscous term for direct and large eddy simulation, Journal of Computational Physics 230 (9) (2011) 3270-3275. doi: $10.1016 / j \cdot j c p .2011 .01 .040$ 
[25] T. Verstraete, S. Scholl, Stability analysis of partitioned methods for predicting conjugate heat transfer, International Journal of Heat and Mass Transfer 101 (2016) 852-869. doi:10.1016/j.ijheatmasstransfer. 2016.05 .041

[26] Z. Wu, D. Laurence, I. Afgan, Direct numerical simulation of a low momentum round jet in channel crossflow, Nuclear Engineering and Design 313 (2017) 273-284. doi:10.1016/j.nucengdes .2016.12.018

[27] T. Dairay, V. Fortuné, E. Lamballais, L. Brizzi, Direct numerical simulation of a turbulent jet impinging on a heated wall, J. Fluid Mech 764 (2015) 362-394. doi:10.1017/jfm.2014.715 